\title{
EMERGENCY LAPAROSCOPIC EXPLORATION OF POSTPARTUM SPONTANEOUS RUPTURE OF THE URINARY BLADDER: A CASE REPORT AND REVIEW OF LITERATURE
}

Zhichao Xu*, Xiaoging Wang*, Jun Wang*, Zhiyan Li*, Junkai Du*

*The First Affiliated Hospital of Xi'an Jiaotong University, Department of Emergency, Xi'an, China

\begin{abstract}
Introduction: Spontaneous rupture of the urinary bladder(SRUB) is a relatively rare cause of acute abdomen disease, which was easily complicated with diffuse peritonitis, septic shock, acute renal failure. although a few cases about postpartum patient sufferred from SRUB have been reported recently, the management of postpartum SRUB was not well established.

Case Report: The subject of this case was a 37-year-old postpartum woman who presented with abdominal pain for one day.Based on his clinical presentation, investigation results, it was highly suspected for urinary disease complicated with diffuse peritonitis, such as SRUB, ureteral rupture. Given that vital signs were unstable and peritonitis signs were aggravating gradually, emergency laparoscopic exploration were performed. There was a visible rupture in the left posterior of the bladder wall, with a diameter of $2 \mathrm{~cm}$. Laparoscopic repair was employed and patient recovered well. In this article, we also reviewed relevant literatures to initially establish main diagnostic basis for postpartum SRUB.
\end{abstract}

Conlcusion:Postpartum women with a complaint of acute abdomen pain maybe caused by SRUB, especially complanied with oliguria and dysuria. Lparoscopy have an obvious advantage to the diagnosis and treatment for highly suspected postpartum SRUB.

Keywords: Spontaneous rupture of the urinary bladder, postpartum, laparoscopy

\section{Introduction}

Spontaneous rupture of the urinary bladder(SRUB) is defined as rupture of the bladder without any trauma or hospital injury, which is gradually recognized by the clinicians. Many causes have been reported are responsible for SRUB, such as bladder tumor, chronic inflammation, bladder diverticulum, neurogenic bladder, excessive alcohal intake, radiocystitis and chemotherapy induced cystitis. While postpartum SRUB without underlying pathology is not well acknowledged and relatively rare in the clinical practice.Postpartum SRUB is mainly onset of acute abdominal pain, abdominal distension ,dysuria, frequently complicated with diffuse peritonitis, septic shock, acute renal failure with the exacerbation of condition. Therefore, it is at most important to give a confirmed diagnosis before occurrence of serious complications.Accordingly, we reported a case of postpartum SRUB diagnosed in our hospital, and reviewed relevant literatures to establish a reasonable management of postpartum SRUB.

\section{Case Report}

A 37-year-old female patient delivered a healthly newborn at 39 weeks of gestation. No episiotomy was performed and revision of birth canal was considered within normal limits. The patient was discharged the following day after she had voided and evacuated. Eight days after vaginal delivery, she presented to the local hospital with a history of acute lower abdominal pain, oliguria and dysuria, without nausea, vomiting and fever. There was only a total of two times of urination, no other obvious incentives contributed to her lower abdominal pain after vaginal delivery. Abdominal ultrasound(US) prompted a large number of abdominopelvic cavity effusion. As it was difficult to disgnosis definitely, she was imminently admitted into our emergency department with a heart rate of 124 beats per minute, respiratory rate $30 / \mathrm{min}$, blood pressure 138/89mmHg.Physical examination revealed abdominal swelling, tenderness, rebound tenderness, shifting dullness, active bowel sounds, no muscle tension. Upon admission, blood routine examination suggested that 
white blood cell $7.72 \times 109 / \mathrm{L}$, neutrophil percentage $91.6 \%$, and urinary routine prompted red blood cells $17126 /$ ul. Renal function prompted creatinine $(\mathrm{Cr})$ 1366umol/L, urea nitrogen(UN) $41.96 \mathrm{mmol} / \mathrm{L}$. Liver function was normal. Diagnostic abdominal puncture tipped light red ascites, and further test prompted acites creatinine 3976 umol/L.Rechecking US prompted mild rough gallbladder wall, kidneys with mild hydronephrosis, postpartum uterus changes, massive fluid in the abdomen and pelvic. Computed Tomography(CT) prompted double full kidneys in morphology, mild hydronephrosis, abdominopelvic cavity effusion. So it was highly suspected for urinary disease, such as SRUB, ureteral rupture. Given that unstable vital signs and peritonitis became aggravated gradually, we decided to perform emergency laparoscopic exploration with the consent of patient and her families. Of intraoperation, a laceration located on the left posterior of the bladder wall was discovered, with a diameter of $2 \mathrm{~cm}$ and a Foley catheter through the laceration. Bladder tumor, stones, and other lesions were not explored (Figure 1). Therefore, laparoscopy repair was carried out. The patient was discharged after 5 days with the catheter closed intermittently. The catheter was removed four weeks later for effective bladder function training, and patient did not complain with any special discomfort by follow-up after $1,3,6$ month.

\section{Dissicusion}

SRUB is a rare cause of abdomianl pain for postpartum women. At present, it is believed that the occurrence of postpartum SRUB is related to unemptying of the bladder and indwelling catheter ineffectively following vaginal delivery, which include many pathophysiologic processes. Firstly, postpartum enlarged and unrecoveried uterus compress urethra, which can weaken the bladder wall gradually; Secondary, compression sufferred from enlarged uterus can directly induce local blood circulation disorders and necrosis of the bladder wall, leading to be necrotic and rupture; Thirdly, it is extensive edema for pelvic and perineal tissue of postpartum patient. Lastly, postpartum abdomian pain can partly cover uresiesthesis. All these factors prone to increase incidence of SRUB. Our patient following vaginal delivery companied with less frequent urination , which did not get attention originally, exacerbated bladder distention and increased the risk of SRUB gradually.

Postpartum SRUB, including intraperitoneal SRUB and extraperitoneal $S R U B$, is mainly on the basis of site of
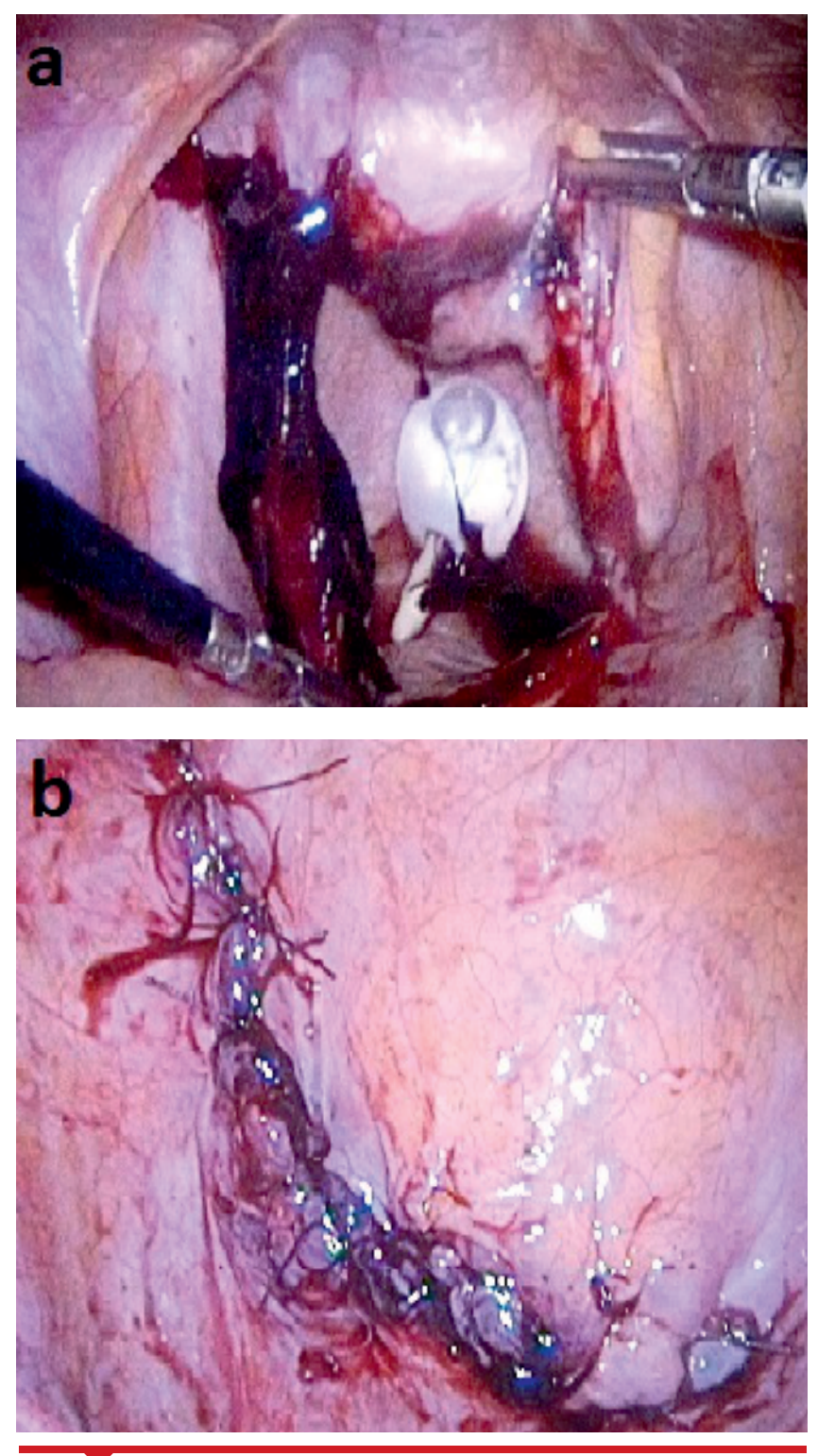

FIGURE 1. A there was a laceration on the left posterior of the bladder wall and a Foley catheter visiable through the laceration. B the laceration was repaired by laparoscopy

rupture. As intraperitoneal SRUB absenced of specific symptoms, it is often ignored and misdiagnosised to acute appendicitis, gastrointestinal perforation, intestinal obstruction. Therefore, we reviewed previous reports(1-8)(Table 1)to further understand diagnostic points of postpartum SRUB. From previous reports, it was suggested that most of postpartum SRUB emerged within 20 days after postpartum, and the most common symptoms were abdominal pain, abdominal distension and dysuria (1-6). Few patients complained with vomiting, fever or hematuria $(7,8)$.Further laboratory examination revealed increased blood $\mathrm{UN}$ and $\mathrm{Cr}(1-6) \cdot \mathrm{In}$ some cases, increased ascites creatinine level could also 
be checked. Most cases could not be diagnosed definitivetly before operation, but a few cases of SRUB was found by cystogram, intravenous pyelogram or vaginal examination $(3,4,7)$. While laparotomy was carried out for suspected postpartum SRUB patients without cystogram. Therefore, SRUB occurred possibly on condition that postpartum patient conformed to following points. Firstly, postpartum patients presented with lower abdominal pain persistently, dysuria, hematuria or signs of peritonitis. Secondly, blood UN and $\mathrm{Cr}$ level increased significantly or ascites $\mathrm{Cr}$ and $\mathrm{UN}$ level increased obviously in light of ascities obtained easily. Lastly, ultrasound reavealed filling bladder and effusion surrounding the bladder. It was essential to perform catheter injection experiments, cystography or surgical exploration directly for patient accorded with one or more points above, which facilitated to discover position of crevasse. In our case, patient complained with abdominal pain, accompanied with hematuria. US and CT suggested mild renal hydronephrosis, massive effusion of abdominopelvic cavity. $\mathrm{Cr}$ of blood and ascites increased significantly. Therefore, our emergency department surgery team highly suspected postpartum SRUB although preoperative examination undiscoveried the site of rupture. With the signs of peritonitis becoming aggravated, emergency laparoscopic exploration was performed, of which SRUB was confirmed and repaired simultaneously.

Generally, it is considerable to take conservative care for patient with small crack, without peritoneal irritation or poor cardiopulmonary function, including indwelling catheter drainage and appropriate antibiotic therapy (9).While conservative treatment is not valid for a majority of patients with peritonitis or extremely unstable vital signs, surgical operation maybe a preferred solution, including hemostasing effectively, repairing gap reliably, aspirating extravasation and flushing thoroughly. In addition, the causes could be remove, including resection of lesion and discharge of obstruction. All these measures can reduce the recurrence possibility of SRUB. With the development and popularity of laparoscopic technique, it is convenient mostly for surgeon to discovery the location and length of concealed cracks, as which is characterized by small incision, less postoperative pain, faster recovery and wider visual field. Therefore, it is irreplaceable to perform comprehensive evaluation by laparoscopy for highly suspected postpartum SRUB.

\section{Conlusion}

Although it is a rare cause of acute abdomen disease, postpartum SRUB is emerged gradually while symptoms of less urination or dysuria induced by different facteros are ingnored. Therefore, it is necessary to keep urination unobstructed following vaginal. And a high index of suspicion is also essential for postpartum women with abdominal pain, abdominal distension, dysuria or hematuria. Lastly, laparoscopic exploration can be employed early to identify and remove causes for suspected postpartum SRUB, which can reduce incidence of fatal complications and give the best possible outcomes.

TABLE 1. Shows the previous reports of postpartum SRUB patient with details of the symptoms, serum $\mathrm{Cr}$, serum $\mathrm{UN}$, ascitic formation, disgnostic means, treatment, site of rupture.

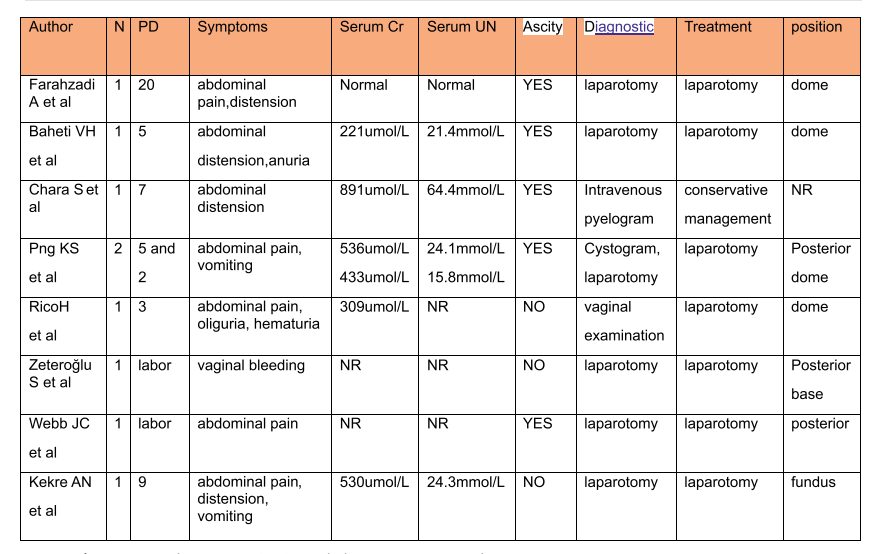

N:Number; PD:Pain Days; Cr:Creatinine; NU:Urea Nitrogen; NR:No

\section{References}

1. Farahzadi A, Mohammadipour S.A Late Presentation of Spontaneous Bladder Rupture During Labor.Urol Case Rep 2016;8:24-5

2. Baheti VH, Wagaskar VG, Patwardhan SK.Missed latrogenic Bladder Rupture Following Normal Vaginal Delivery.J Clin Diagn Res 2015;9:PD01-2

3. Charalampidis S, Petrides C, Charalampous C, Stavrou S.Hyponatremic renal pseudofailure and massive ascites following normal vaginal delivery: a diagnostic and therapeutic challenge.Ren Fail 2012;34:237-40.

4. Png KS, Chong YL, Ng CK.Two cases of intraperitoneal bladder rupture following vaginal delivery.Singapore Med J 2008;49:e327-9.

5. Rico H,Dueñas-García OF,Gorbea-Sanchez V,Herrerias-canedo T.Bladder rupture caused by postpartum urinary retention.Obstet Gynecol 2008;112:481-2.

6. Zeteroğlu S, Bayrakli H, Yilmaz Y, Sahin HG.Combined bladder, 
urethral and uterine rupture after a normal, spontaneous vaginal delivery at home: a case report.J Reprod Med 2005;50:874-6.

7. Webb JC, Gilson G, Gordon L.Late second stage rupture of the uterus and bladder with vaginal birth after cesarean section: a case report and review of the literature.J Matern Fetal Med 2000;9:362-5.

8. Kekre AN, Kekre N, Nath V,Seshadri L.Spontaneous rupture of the urinary bladder in the puerperium.Aust N Z J Obstet Gynaecol 1997;37:473-4.

9. Abu Mahfouz I,Sayer T,Phillips C.Conservative management of spontaneous rupture of the urinarybladder.IntUrogynecol J 2011;22:629-3 